\title{
Robustness analysis of biomolecular networks
}

\author{
Steffen Waldherr
}

\begin{abstract}
Robustness is a widely studied property of biomolecular networks, and control engineers are in a good position to make significant contributions here. This tutorial defines common robustness analysis problems for biomolecular networks, presents computational methods to perform the analysis, and shows by means of examples what can be learned about a network from the analysis results. The focus is on deterministic methods applied to ordinary differential equation models of biomolecular networks.
\end{abstract}

\section{INTRODUCTION}

"Biological systems are robust" is an observation that everybody makes almost everyday, mostly by physical experience with one's own body. We are able to deliver conference talks despite viral infections, or move on after stumbling or falling. How does this everyday observation relate do the deeper foundations of life, such as the biomolecular networks that process energy and information in living cells? That is one of the driving questions for robustness analysis of biomolecular networks, a field with high research activity and significant contributions from the control community over the past 15 years.

There are many well studied examples for robustness in biochemical networks. Circadian clocks in a wide range of organisms are robust time-keepers despite environmental variations, most notably different temperature levels [25], and internal perturbations such as variations in gene expression levels [8]. Another example is the chemotactic system which allows bacteria to react to environmental chemicals, showing a remarkable robustness against variations in gene expression level of its molecular components [1]. Robustness analysis by means of mathematical models is therefore an important research topic in biomolecular network analysis.

This tutorial is going to discuss three questions related to robustness analysis in the context of biomolecular networks: what is robustness, how to analyze it, and how to interpret the results in a biological context. Section II summarizes robustness definitions which are relevant for biomolecular networks, and outlines specific challenges for robustness analysis in this context. Section III gives an overview on existing methods to compute robustness measures based on a mathematical model of the network. Section IV shows by means of examples which insights into biology can be gained from a robustness analysis.

Due to the wide range of robustness concepts and computational approaches even in the focussed area of biomolecular

\footnotetext{
*This work was supported by the German federal state Sachsen-Anhalt through the Center for Dynamical Systems - Biosystems Engineering.

Steffen Waldherr is with the Institute for Automation Engineering, Otto-von-Guericke-University, $39106 \quad$ Magdeburg, Germany steffen.waldherreovgu. de
}

networks, the scope of this tutorial inevitably needs to be narrowed to few specific concepts. The following limitations apply:

- We are looking at models described by ordinary differential equations (ODEs).

- We consider robustness analysis methods taking only the mathematical model, a perturbation class, and a desired behaviour into account.

- We exclude probabilistic analysis methods, for example based on Monte Carlo sampling.

These limitations exclude other important modeling frameworks, such as discrete (or logical) models [27] and stochastic models [18]. Also approaches where robustness analysis is coupled with experimental data, for example to perform model validation or invalidation, are not in the scope of this tutorial. For results in this direction and robustness measures in the context of parameter estimation, the reader is referred to the recent review [32].

\section{DEFINING ROBUSTNESS IN BIOMOLECULAR NETWORKS}

This section summarizes relevant definitions of robustness in ODE models of biomolecular networks, and outlines the challenges resulting from these definitions. At its core, robustness means the ability of a system to maintain its function (in a sense of purpose, not mathematically) despite perturbations. Any formal robustness definition therefore requires three components: the system to be considered, the function that the system is to maintain, and the class of perturbations acting on the system. In classical robust control, the system is an LTI system, the function to be maintained is asymptotic stability of the origin, and the perturbation class is another, uncertain LTI system $\Delta$ that is connected to the nominal system [40].

1) Definition of the system: For this tutorial, we consider robustness problems where the system is a model for a biomolecular network, given by the ODE

$$
\dot{x}=S v(x),
$$

where $x \in \mathbb{R}^{n}$ is a vector of biochemical concentrations, $S \in \mathbb{R}^{n \times m}$ is the stoichiometric matrix, and $v(x) \in \mathbb{R}^{m}$ is the concentration-dependent reaction rate vector, together with the initial condition

$$
x(0)=x_{0} .
$$

A challenge that already arises from the choice of system is that reaction rates are commonly non-linear functions of the concentrations, which calls for the application of analysis methods that are able to cope with this non-linearity [11]. 
2) Definition of the perturbation class: Perturbations to consider for robustness analysis of this model are changes in the right hand side of the differential equation (1) or in the initial condition (2). We distinguish parametric, nonparametric, and structural perturbations. In many biomolecular network models, the reaction rate vector depends on additional model parameters, called the reaction rate constants, such that the differential equation becomes

$$
\dot{x}=S v(x, \mu)
$$

where $\mu$ is a vector of reaction rate constants. These parameter values vary for example with temperature or other environmental conditions [5], and robustness against parametric perturbations is thus a commonly considered problem for biomolecular networks [24]. Other parameters may for example represent the total expression level of proteins, where slow stochastic fluctuations give rise to significant variability. Parameters can also be uncertain as a result of the modeling process, for example when identifying parameter values from noisy experimental data. The perturbation class is often formalized as lower and upper bounds on the parameter values, i.e., one considers a set given by

$$
\mathcal{P}=\left\{\mu \mid \mu_{\min } \leq \mu \leq \mu_{\max }\right\}
$$

where $\mu_{\min }, \mu_{\max }$ are vectors of element-wise bounds.

In some cases, it may also be relevant to consider variations in the initial condition $x_{0}$. This concerns networks containing conservation relations [13], for example total enzyme amounts which are not consumed in the network. Uncertainty in the initial condition has for example been used in the analysis of concentration robustness [28], [31].

Perturbations in parameters or initial conditions are appropriate to consider when the reaction mechanism and the corresponding mathematical expression for the reaction rate are well-defined. However, this is often not the case, especially when several reaction steps are combined into a single overall reaction. Then, it becomes appropriate to consider non-parametric perturbations, which are defined as arbitrary variations in the reaction rate expressions $v(x)$. This approach has been pursued in [35], where reaction rate functions are considered to vary from the slope of nominal reaction rates $v_{n o m}$, at some predefined point $\bar{x}$. Such a perturbation class can be defined by

$$
\mathcal{P}=\left\{v \in \mathcal{C}^{1}\left(\mathbb{R}^{n}\right) \mid \frac{\partial v}{\partial x}(\bar{x})-\frac{\partial v_{\text {nom }}}{\partial x}(\bar{x}) \in \boldsymbol{\Delta}\right\},
$$

where $\boldsymbol{\Delta} \subset \mathbb{R}^{m \times n}$ is an appropriate set of admissible perturbations to the reaction rate Jacobian. Perturbations of this class have been denoted as kinetic perturbations in [35]. Conceptually, such non-parametric variations are related to the perturbations considered in the structural stability of nonlinear systems [20], [9], albeit they still respect the network structure imposed by the stoichiometric matrix $S$.

Another type of non-parametric perturbations can be considered when the dynamics of the network can be decomposed as a linear system with a non-linear static feedback.
The ODE would then be in so-called Lure form, given by

$$
\dot{x}=A x+B \Phi(C x),
$$

with uncertainty in the feedback function $\Phi$. This description is especially relevant for genetic networks, where transcription and translation are modelled with linear dynamics, and only the activity of transcription factors is modelled as a static non-linearity [7]. The perturbation class is then defined by restrictions on the function $\Phi$. In the spirit of absolute stability theory, it is common to use a sector constraint on the non-linearity $\Phi$ [21], given for example by

$$
\Phi^{\mathrm{T}}(y)(\Phi(y)-K y) \leq 0 .
$$

Furthermore, one can consider structural perturbations, where the interaction structure of the network is being varied. Such perturbations could be due to changes in the stoichiometric matrix $S$, which may arise from gene mutations or from enzyme knockouts in metabolic networks [38]. Another possibility is to model arbitrary, even dynamic perturbations within the interaction structure, for example by perturbing some elements of the network's Jacobian $A(x)=S \frac{\partial v}{\partial x}$ [17], [33].

3) Definition of the desired functionality: Finally, let us consider how to define the system's functionality that should be maintained despite perturbations. In the framework applied here, the functionality of the system will be determined by properties of the solution $x(t)$ of the differential equation (1) with initial condition (2). We can say that the system maintains its functionality, if the solutions $x(t)$ as a trajectory satisfies $x(\cdot) \in \mathcal{F}$ for any perturbation within the given class, where $\mathcal{F}$ is the set of trajectories where the desired functionality is present. How this $\mathcal{F}$ is defined then depends on the biological question under consideration.

Before looking at more specific examples of relevant biological functionality, let us first formulate two generic robustness analysis problems based on the three aspects discussed above - the system, the perturbation class, and the desired functionality. The first problem is simply to check whether the system's functionality is robust against perturbations from the given class, with a yes/no result. This can be formalized as follows.

Problem 1: Given an ODE model (1) with initial condition (2), a perturbation class $\mathcal{P}$ and a set of trajectories $\mathcal{F}$ representing the desired functionality, determine whether solutions $x(t)$ of the model (1) satisfy $x(\cdot) \in \mathcal{F}$ for all perturbations in $\mathcal{P}$.

One may also aim to get a more quantitative measure of a system's robustness. Given a measure for a perturbation class, we may define a robustness measure as follows [34].

Problem 2: Given an ODE model (1) with initial condition (2), a set of measurable perturbation classes and a set of trajectories $\mathcal{F}$ representing the desired functionality, determine the largest perturbation class $\mathcal{P}$ such that solutions $x(t)$ of the model (1) satisfy $x(\cdot) \in \mathcal{F}$ for all perturbations in $\mathcal{P}$.

This robustness measure is illustrated in Figure 1. Alternatively, if we don't have a measure for perturbation 


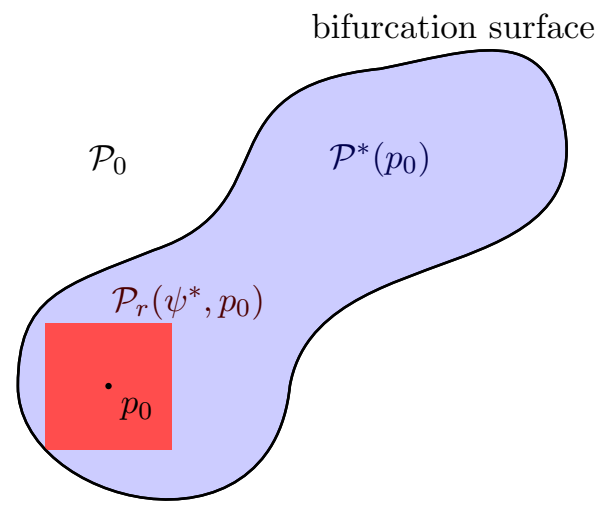

Fig. 1. Illustration of robustness measure in perturbation space. The system maintains its functionality within the blue area. The perturbation class are cubes around a nominal point, where the measure of a cube is defined by its radius. The robustness measure of the system is the largest such cube that fits inside the blue area. Reproduced from [34] with permission from Elsevier.

classes, but do have one for single perturbations, a robustness measure may also be defined as the size of the smallest perturbation in the considered perturbation class $\mathcal{P}$ that makes the system loose its functionality.

Let us now consider more specifically the types of a system's functionality that are commonly considered in the area of biomolecular networks. In many signalling and gene regulation networks, the core functionality is related to the qualitative dynamic properties of the system. Dynamic properties which have been considered include sustained oscillations [33], bistability [10], or perfect adaptation [1]. Since changes in oscillations or stability properties under parameter variations are due to bifurcations in the considered system, bifurcation theory plays a prominent role in robustness analysis, when considering these types of functionality together with parametric perturbations in a non-linear model [24], [22], [34].

While sustained oscillations or bistability are structurally stable dynamic properties [20], the property of showing perfect adaptation is not generic. Thus robustness of perfect adaptation against parametric perturbations is tied to specific structural features of the network, which will be discussed in more detail in Section IV.

While the exact concentration values may not be relevant for the functionality of some networks, they will certainly play an important role for others. In previous studies, commonly the steady states of a subset of concentration values have been related to the system's functionality. For example, in a fine-tuned signalling pathway, the exact concentration value of the downstream effector would be relevant, but not the values of intermediate elements. A specific example are two-component signalling pathways, where only the concentration of the active response regulator is relevant for functionality, but for example not the concentration of the intermediate receptor [3], [31]. The associated robustness analysis problem has been termed concentration robustness
[28] and has been studied with both perturbations in the initial condition [28] and with more general parametric perturbations [31].

While the types of functionality discussed so far are relevant for a wide range of biomolecular networks, more specific cases may require a more specific definition of functionality. To give just one example: for oscillating networks such as the circadian clock, not only the existence of oscillations will be relevant, but also their exact period [30].

\section{METHODS FOR MODEL-BASED ROBUSTNESS ANALYSIS}

\section{A. Dynamic robustness analysis with parametric perturba- tions}

Let us first discuss the robustness analysis problem with parametric perturbations, where the system under consideration is a parametrized ODE as in (3), with the desired functionality given by the qualitative dynamic behaviour.

As discussed above, the robustness of the qualitative dynamic behaviour is tightly linked to bifurcations occurring upon parameter variations. Ideally, a complete bifurcation diagram for the model would be available, and robustness analysis could be performed straightforward based on that diagram. A related definition of a robustness measure with a scalar parameter $\mu>0$ is the degree of robustness defined by Ma \& Iglesias [22], given by

$$
\operatorname{DOR}\left(\mu_{0}\right)=1-\max \left\{\frac{\check{\mu}}{\mu_{0}}, \frac{\mu_{0}}{\hat{\mu}}\right\},
$$

where $\check{\mu}$ and $\hat{\mu}$ are the closest bifurcation points smaller and larger than the nominal parameter value $\mu_{0}$, respectively. The robustness measure $D O R$ guarantees that no bifurcations, and therefore no loss of functionality related to the qualitative dynamic properties, will occur for any multiplicative parameter perturbation less than $(1-D O R)^{-1}$.

A similar robustness measure can be defined for multiparametric systems by considering a hyperrectangle around the nominal parameter vector $\mu_{0}$ as follows [34]. First, define a hyperrectangle $\mathcal{P}$ in parameter space as

$$
\mathcal{P}\left(\psi, \mu_{0}\right)=\left\{\mu \mid \frac{1}{\psi} \leq \frac{\mu}{\mu_{0}} \leq \psi\right\},
$$

where the inequality is considered to be element-wise. Then a robustness measure $\psi^{*}$ for a nominal point $\mu_{0}$ is given by

$\psi^{*}\left(\mu_{0}\right)=\sup \left\{\psi \geq 1 \mid \mathcal{P}\left(\psi, \mu_{0}\right)\right.$ contains no bifurcations $\}$.

Unfortunately, bifurcation diagrams for most models of biomolecular networks can only be computed numerically, and the commonly applied continuation methods are limited to a single or few parameters to vary [20], [14]. Thus, obtaining complete bifurcation diagrams for models with more than a few parameters is usually not possible, and even with a single parameter, continuation methods can not guarantee that all bifurcation points are found, as some might be disconnected from the chosen starting point in the state/parameter space. Computational robustness analysis therefore focusses on estimating lower and upper bounds 
on the robustness measures given in (8) or (10). This is particularly successful when restricting the bifurcations to bifurcations of equilibrium points, excluding for example bifurcations of limit cycles. In that case, one can check robustness by studying the dynamic properties of linear approximations around the system's equilibrium points, and the robustness measure (10) now becomes [34]

$$
\begin{array}{r}
\psi^{*}\left(\mu_{0}\right)=\sup \left\{\psi \geq 1 \mid \forall \mu \in \mathcal{P}\left(\psi, \mu_{0}\right) \forall x \in \mathbb{R}^{n}:\right. \\
\left.S v(x, \mu)=0 \Rightarrow \sigma\left(S \frac{\partial v}{\partial x}(x, \mu)\right) \cap \mathbf{j} \mathbb{R}=\emptyset\right\},
\end{array}
$$

where $\sigma(A)$ is the spectrum of the matrix $A$. Thus, while the problem is still non-linear due to the non-linear dependence of $v$ and its Jacobian on $x$ and $\mu$, we are now much closer to classical robust control theory, in that a property of an uncertain matrix' spectrum is at the core of this robustness analysis problem. A remaining challenge is the non-linear equality constraint $S v(x, \mu)=0$. In common biomolecular modelling approaches, the reaction rates $v(x, \mu)$ are given as polynomial or rational functions. Thus, polynomial programming methods have successfully been applied to compute a robustness measure in this case [11], [34].

\section{B. Steady state robustness analysis with parametric pertur- bations}

Let us next consider the problem of concentration robustness, where the functionality of the system is tied to the steady state values of certain output variables, under parametric perturbations. In many biomolecular networks, the relevant outputs are linear functions of the concentration variables, given by

$$
y=C x,
$$

with $C \in \mathbb{R}^{q \times n}$. We will focus on the special case where the output variables are actually a subset of the concentration variables, i.e., $C$ is of the form

$$
C=\left(e_{i}^{\mathrm{T}}\right)_{i \in \mathcal{I}_{y}},
$$

where $\mathcal{I}_{y} \subset\{1,2, \ldots, n\}$ are the indices of the concentration variables appearing in the output, and $e_{i}$ is the $i$-th basis vector in $\mathbb{R}^{n}$.

Under certain conditions on the network related to the implicit function theorem, which are generically satisfied, we can define a region $\mathcal{P}$ in parameter space where the function $k: \mathcal{P} \rightarrow \mathbb{R}^{n}$ defines a parameter-dependent steady state of the network, i.e.,

$$
S v(k(\mu), \mu)=0
$$

for $\mu \in \mathcal{P}$. Robustness of the steady state output concentrations is then achieved if the steady state output $C k(\mu)$ is independent of the parameters $\mu$, or, equivalently,

$$
C \frac{\partial k}{\partial \mu}(\mu)=0
$$

for $\mu \in \mathcal{P}$ [31].

A question of particular biological relevance is under which conditions on the network structure we get steady state output robustness independent of the specific reaction kinetics $v(x, \mu)$, but only based on structural information encoded in the stoichiometric matrix $S$ and in the zero pattern of the Jacobian of $v$. A sufficient and necessary condition for steady state output robustness for the special case of an output as in (13) has been proposed in [31]. For simplicity, we only discuss the case of biomolecular networks with a scalar parameter and without conservation relations here, albeit the result in [31] is more general. Define the matrix $M$ as

$$
M(\mu)=\operatorname{dg}(v(k(\mu), \mu))^{-1} \frac{\partial v}{\partial x}(k(\mu), \mu) \operatorname{dg} k(\mu) C^{\mathrm{T}},
$$

where $\operatorname{dg} v$ denotes a diagonal matrix with the elements of the vector $v$ on its diagonal, and the vector $P$ as

$$
P(\mu)=\operatorname{dg}(v(k(\mu), \mu))^{-1} \frac{\partial v}{\partial \mu}(k(\mu), \mu) \mu .
$$

Then steady state output robustness can be characterized as follows.

Theorem 1 ([31]): The network has steady state output robustness, if and only if

$$
P(\mu) \in \operatorname{im} M(\mu)+\operatorname{ker}(S \operatorname{dg} v(k(\mu), \mu))
$$

for all $\mu \in \mathcal{P}$.

In networks where reactions are modelled by mass action or generalized mass action, i.e., $v_{j}(x, \mu)=k_{j}(\mu) \prod_{i=1}^{n} x_{i}^{\alpha_{i j}}$ with constant real exponents $\alpha_{i j}$, condition (18) can be formulated independently of the parameter $\mu$ and can thus be checked by standard linear algebra tools.

The condition (18) asserts steady state output robustness under the condition that the network's steady state $k(\mu)$ does not undergo any bifurcations for $\mu \in \mathcal{P}$. This assumption can be checked for example by the robustness analysis approach discussed in Section III-A.

Another approach to steady state output robustness has been taken in [28]. Based on mass action network theory [12], they provide a sufficient condition for steady state output robustness for the special case of mass action networks and perturbed parameters being concentrations of conserved chemical species (termed absolute concentration robustness). While the result is restricted to a smaller system class than the one presented in [31], an advantage is that the condition in [28] directly guarantees stability of the steady state, and a further dynamical robustness analysis is not necessary.

\section{Robustness analysis with non-parametric perturbations}

Let us next discuss the robustness analysis in the case of non-parametric perturbations.

With kinetic perturbations as defined in (5), linear robust control theory has been applied to check robustness of the qualitative dynamical behaviour, i.e., to exclude the occurrence of local bifurcations [35]. For the perturbation class given by (5), the Jacobian of the network ODE's right hand side is evaluated at a steady state $\bar{x}$ to yield

$$
\tilde{A}_{\bar{x}}(\Delta)=S\left(\frac{\partial v}{\partial x}(\bar{x})+\Delta\right),
$$


where $\Delta$ is a real $m \times n$ matrix satisfying the constraint $\Delta \in \Delta$ imposed by the perturbation class (5). The robustness analysis problem 1 then consists in checking that the matrix $A_{\bar{x}}(\Delta)$ does not have an eigenvalue on the imaginary axis for any admissible $\Delta$, i.e., one needs to check the condition

$$
\operatorname{det}\left(I_{m}-\Delta G(\mathbf{j} \omega)\right) \neq 0
$$

is true for any $\omega \geq 0$, where the transfer matrix $G(\mathbf{j} \omega)$ is defined as

$$
G(\mathbf{j} \omega)=\left(\mathbf{j} \omega I_{n}-A\right)^{-1} S .
$$

Using robust control theory, it is even possible to tackle the robustness analysis problem 2 , i.e., to compute a robustness measure for the dynamical behaviour under kinetic perturbations. Consider the structured singular value [15] defined by

$$
\begin{aligned}
\mu_{\boldsymbol{\Delta}}(G(\mathbf{j} \omega))= & (\inf \{\|\Delta\| \mid \Delta \in \boldsymbol{\Delta}, \\
& \left.\left.\operatorname{det}\left(I_{m}-\Delta G(\mathbf{j} \omega)\right)=0\right\}\right)^{-1},
\end{aligned}
$$

with a suitable matrix norm $\|\cdot\|$. A robustness measure is then given by

$$
R=\left(\sup _{\omega} \mu_{\Delta}(G(\mathbf{j} \omega))\right)^{-1},
$$

where $R$ is the largest value such that the constraint $\|\Delta\|<$ $R$ on the perturbation $\Delta$ guarantees robustness. Usually only lower and upper bounds on the structured singular value can be computed [40], [15]. An upper bound on $\mu$ can be used to compute a lower bound on the robustness measure.

For non-parametric perturbations with a static non-linear feedback as in (6), absolute stability theory can be used to check for robust stability [21]. This approach has been extended recently towards instability analysis [16], thus extending the methodology towards more general types of dynamic behaviour. The methods are based on LMIs and analysis of system gains, and can be applied to solve the robustness analysis problems 1 and 2 .

\section{APPLICATIONS IN BIOMOLECULAR NETWORKS}

Which insights into biology can be gained from robustness analysis? That is the question to be approached in this section.

Let us first focus on robustness analysis of the steady state output concentration. This is closely related to adaptive behaviour of biomolecular networks, which has found significant attention in the biological literature over the past 15 years [2], [1], [23]. From a systems-theoretic perspective, perfect adaptation can be defined by a zero steady state gain [37], i.e., the response returns to the pre-stimulus value after a sufficiently long time. A range of biological signalling systems have adaptation as key element of their functionality. Looking for example at chemotaxis, this property is important in order to maintain sensitivity of the signalling system towards concentration gradients for a wide range of basal concentration levels [6].

Robustness of adaptation is then relevant as it means that adaptation is maintained despite for example variations in intracellular protein concentrations. Based on control theoretic
A

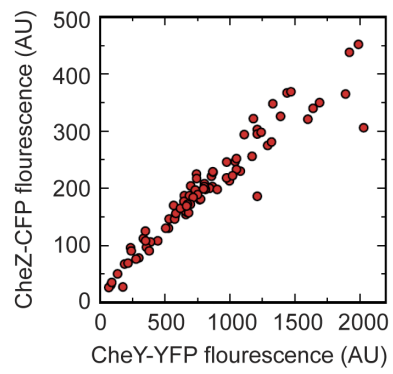

B

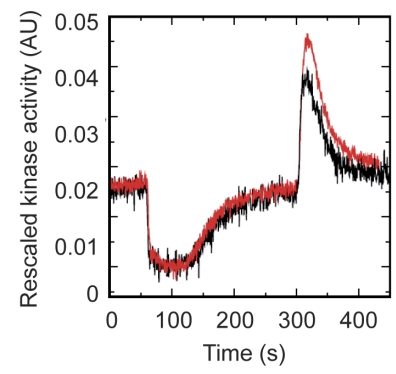

Fig. 2. Experimental validation of concentration robustness in the E. coli chemotaxis pathway. (A) Biological variability of protein expression levels. (B) Stimulus-response dynamics of native (black) and seven fold upregulated (red) pathway. Reproduced from [31].

approaches, it has become clear that integral feedback [39], or, more generally, an internal model of the stimulus [29], is required for robust adaptation.

Adaptation is a special case of the steady state output robustness discussed above. Thus, insight into biological network properties related to adaptation could also be gained from the corresponding methods discussed in Section IIIB. For example, the robustness analysis of the chemotactic pathway performed in [31] revealed that not only the structure of the signalling network, but also its genomic organization contributes to the robustness of adaptation. The analysis showed that adaptation is robust if perturbations in total protein expression levels are proportional (Figure 2A), which is assured in cells by having the corresponding genes on the same operon. This property of the pathway contributes to robustness of adaptation over a wide range of perturbations (Figure 2B).

Next, let us discuss applications for the robustness analysis of the dynamic behaviour of biomolecular networks, especially oscillations and bistability. This type of robustness analysis has mostly been applied in order to validate or invalidate models of biochemical signalling networks or genetic regulatory networks [4]. With this approach, it is particularly relevant to detect non-robust perturbations for the models: if the corresponding perturbation is plausible in the corresponding biological system, then such a result indicates shortcomings and potentially lacking mechanisms in the model. Here, robustness analysis is useful to refine models of the considered networks and to extend the associated biological knowlegde.

A robustness analysis of bistability in the GAL regulatory system has been reported in [26]. The authors use an LMI characterization based on the bioSDP toolbox [36] to assert the existence of two distinct domains of attraction under parametric uncertainty. They established robust bistability for parameter uncertainties of up to $20 \%$, thus validating the model with respect to its bistability property.

Another example concerns the robustness analysis of sustained oscillations in the central metabolism of activated neutrophils [17]. This study used non-parametric perturbations similar to the ones discussed in Section III-C, revealing that 
the existing model is very fragile to dynamic perturbations. A later study of the same model then suggested an extension to the model involving Calcium signalling, which served to significantly increase robustness of the oscillations [19].

\section{Concluding nOte}

Models, especially on the intracellular level in biology, often involve significant uncertainties, and it is important to distinguish robustness analysis with respect to model uncertainties from actual perturbations acting on the system. For systems where models are well validated, such as bacterial chemotaxis, robustness analysis can be used to learn something about the system. But even if the model is not sufficiently well validated, robustness analysis is useful, since it will either show where the model has weaknesses, or alternatively assert that the model reproduces the system's behaviour robustly.

\section{ACKNOWLEDGMENTS}

I thank Philipp Rumschinski and Stefan Streif for helpful comments on an earlier version of this paper.

\section{REFERENCES}

[1] U. Alon, M. G. Surette, N. Barkai, and S. Leibler. Robustness in bacterial chemotaxis. Nature, 397(6715):168-171, Jan 1999.

[2] N. Barkai and S. Leibler. Robustness in simple biochemical networks. Nature, 387(6636):913-917, Jun 1997.

[3] E. Batchelor and M. Goulian. Robustness and the cycle of phosphorylation and dephosphorylation in a two-component regulatory system. Proc. Natl. Acad. Sci. U.S.A., 100(2):691-696, Jan 2003.

[4] D. G. Bates and C. Cosentino. Validation and invalidation of systems biology models using robustness analysis. IET Syst. Biol., 5(4):229244, 2011.

[5] D. A. Beard and H. Qian. Chemical Biophysics: Quantitative Analysis of Cellular Systems. Cambridge University Press, 2006.

[6] D. Bray. Bacterial chemotaxis and the question of gain. Proc. Natl. Acad. Sci. U.S.A., 99(1):7-9, Jan 2002.

[7] L. Chen and K. Aihara. A model of periodic oscillation for genetic regulatory systems. IEEE Trans. Circuits Syst. I, 49(10):1429-1436, 2002.

[8] S. Clodong, U. Dühring, L. Kronk, A. Wilde, I. Axmann, H. Herzel, and M. Kollmann. Functioning and robustness of a bacterial circadian clock. Mol. Syst. Biol, 3:90, 2007.

[9] F. Cordoleani, D. Nerini, M. Gauduchon, A. Morozov, and J.-C. Poggiale. Structural sensitivity of biological models revisited. J. Theor. Biol., 283(1):82-91, Aug 2011.

[10] T. Eissing, F. Allgöwer, and E. Bullinger. Robustness properties of apoptosis models with respect to parameter variations and intrinsic noise. IEE P. Syst. Biol., 152(4):221-228, December 2005.

[11] H. El-Samad, S. Prajna, A. Papachristodoulou, J. Doyle, and M. Khammash. Advanced methods and algorithms for biological networks analysis. Proc. of the IEEE, 94(4):832-853, 2006.

[12] M. Feinberg. Chemical reaction network structure and the stability of complex isothermal reactors - I. The deficiency zero and deficiency one theorems. Chem. Eng. Sci., 42:2229-68, 1987.

[13] R. Heinrich and S. Schuster. The Regulation of Cellular Systems. Chapman \& Hall, New York, 1996.

[14] M. E. Henderson. Higher-dimensional continuation. In B. Krauskopf, editor, Numerical continuation methods for dynamical systems, pages 77-115. Springer, Dordrecht, 2007.

[15] D. Hinrichsen and A. J. Pritchard. Mathematical Systems Theory I. Springer-Verlag, Berlin, 2005.

[16] M. Inoue, J.-I. Imura, K. Kashima, T. Arai, and K. Aihara. An instability condition for uncertain systems toward robust bifurcation analysis. In Proc. of the European Control Conference (ECC), pages 3264-3269, Zürich, Switzerland, 2013.
[17] E. W. Jacobsen and G. Cedersund. Structural robustness of biochemical network models-with application to the oscillatory metabolism of activated neutrophils. IET Syst. Biol., 2(1):39-47, Jan 2008.

[18] M. Khammash and H. El Samad. Stochastic Modeling and Analysis of Genetic Networks. In Proc. of the 44th IEEE Conf. on Dec. and Control, Sevilla, Spain, 2005.

[19] J.-S. Kim, N. V. Valeyev, I. Postlethwaite, P. Heslop-Harrison, K.H. Cho, and D. G. Bates. Analysis and extension of a biochemical network model using robust control theory. Int. J. Robust Nonlinear Control, 20:1017-1026, 2010.

[20] Y. A. Kuznetsov. Elements of Applied Bifurcation Theory. SpringerVerlag, New York, 1995.

[21] C. Li, L. Chen, and K. Aihara. Stability of genetic networks with SUM regulatory logic: Lur'e system and LMI approach. IEEE Trans. Circ. Syst. I, 53(11):2451-2458, 2006.

[22] L. Ma and P. A. Iglesias. Quantifying robustness of biochemical network models. BMC Bioinf., 3:38, 2002.

[23] W. Ma, A. Trusina, H. El-Samad, W. A. Lim, and C. Tang. Defining network topologies that can achieve biochemical adaptation. Cell, 138(4):760-773, Aug 2009.

[24] M. Morohashi, A. E. Winn, M. T. Borisuk, H. Bolouri, J. Doyle, and $\mathrm{H}$. Kitano. Robustness as a measure of plausibility in models of biochemical networks. J. Theor. Biol., 216(1):19-30, May 2002.

[25] D. A. Rand, B. V. Shulgin, J. D. Salazar, and A. J. Millar. Uncovering the design principles of circadian clocks: mathematical analysis of flexibility and evolutionary goals. J. Theor. Biol., 238(3):616-635, Feb 2006.

[26] L. Salerno, C. Cosentino, A. Merola, D. G. Bates, and F. Amato. Validation of a model of the gal regulatory system via robustness analysis of its bistability characteristics. BMC Syst. Biol., 7:39, 2013.

[27] R. Samaga and S. Klamt. Modeling approaches for qualitative and semi-quantitative analysis of cellular signaling networks. Cell Commun Signal., 11(1):43, 2013.

[28] G. Shinar and M. Feinberg. Structural sources of robustness in biochemical reaction networks. Science, 327(5971):1389-1391, Mar 2010.

[29] E. D. Sontag. Adaptation and regulation with signal detection implies internal model. Syst. Control Lett., 50:119-126, 2003.

[30] J. Stelling, E. D. Gilles, and F. J. Doyle III. Robustness properties of circadian clock architectures. Proc. Natl. Acad. Sci. U.S.A., 101(36):13210-13215, Sep 2004.

[31] R. Steuer, S. Waldherr, V. Sourjik, and M. Kollmann. Robust signal processing in living cells. PLoS Comput. Biol., 7(11):e1002218, November 2011.

[32] S. Streif, K.-K. K. Kim, P. Rumschinski, M. Kishida, D. E. Shen, R. Findeisen, and R. D. Braatz. Robustness analysis, prediction and estimation for uncertain biochemical networks. In Proc. 10th IFAC Int. Symp. Dyn. Contr. Proc. Syst. (DYCOPS), pages 1-20, Mumbai, India, Dec 18-20 2013. Invited plenary paper.

[33] C. Trané and E. W. Jacobsen. Network structure and robustness of intracellular oscillators. In Proc. of the 17th IFAC World Congress, Seoul, Korea, pages 10989-94, 2008.

[34] S. Waldherr and F. Allgöwer. Robust stability and instability of biochemical networks with parametric uncertainty. Automatica, 47:11391146, 2011.

[35] S. Waldherr, F. Allgöwer, and E. W. Jacobsen. Kinetic perturbations as robustness analysis tool for biochemical reaction networks. In Proc. of the 48th IEEE Conf. on Dec. and Control, Shanghai, China, pages 4572-4577, 2009.

[36] S. Waldherr, J. Hasenauer, and Frank Allgöwer. Set based uncertainty analysis and parameter estimation of biological networks with the bioSDP toolbox. In Proc. of the 9th Intern. Workshop Comp. Syst. Biol. (WCSB), Ulm, Germany, 2012.

[37] S. Waldherr, S. Streif, and F. Allgöwer. Design of biomolecular network modifications to achieve adaptation. IET Syst. Biol., 6:223231, December 2012.

[38] T. Wilhelm, J. Behre, and S. Schuster. Analysis of structural robustness of metabolic networks. Syst. Biol. (IEE), 1(1):114-120, 2004.

[39] T. M. Yi, Y. Huang, M. I. Simon, and J. Doyle. Robust perfect adaptation in bacterial chemotaxis through integral feedback control. Proc. Natl. Acad. Sci. U.S.A., 97(9):4649-53, Apr 2000.

[40] K. Zhou, J. C. Doyle, and K. Glover. Robust and Optimal Control. Prentice Hall, Upper Saddle River, New Jersey, 1996. 